\title{
An Approach for System Identification in Unmanned Surface Vehicles
}

\author{
Izzet Kagan Erunsal $^{1 *}$, Kenan Ahiska ${ }^{2}$, Murat Kumru ${ }^{3}$, Mehmet Kemal Leblebicioglu ${ }^{4}$ \\ ${ }^{1}$ Aselsan Inc., 06370 Yenimahalle, Ankara, Turkey (kerunsal@aselsan.com.tr) * Corresponding author \\ ${ }^{2}$ Aselsan Inc., 06370 Yenimahalle, Ankara, Turkey (kahiska@aselsan.com.tr) \\ ${ }^{3}$ Electrical and Electronics Engineering Dpt., Middle East Technical University, \\ 06800, Cankaya, Ankara, Turkey (kumru@metu.edu.tr) \\ ${ }^{4}$ Electrical and Electronics Engineering Dpt., Middle East Technical University, \\ 06800, Cankaya, Ankara, Turkey (kleb@metu.edu.tr)
}

\begin{abstract}
In this study, a system identification methodology is introduced to determine the model parameters of unmanned surface vehicles. The proposed identification scheme is based on sequencing the experiments according to their capabilities to identify the model parameters. In each experiment, the parameters to be found are updated and the results are validated before ascertaining the final value. A procedure to complete the identification work in an experiment, namely the required post-processing, the optimization routines, the cost function evaluations are defined and discussed. The final parameter set is validated in random motion tests with rich motion content. It has been observed that the proposed method elicits a parameter identification with remarkable success.
\end{abstract}

Keywords: Unmanned surface vehicles, system identification, parameter estimation

\section{INTRODUCTION}

Unmanned surface vehicles (USV) have been proposed and utilized to protect valuable human resources from risky and complicated missions. Efficient autopilot designs for USVs require accurate models [1]. In this study, a 6-DoF (degree of freedom) mathematical model for USVs is constructed, and a systematic methodology is introduced to identify the model parameters. The system identification process for USVs will generate a foundation for better understanding of the vessel motion, and it will provide a valuable insight for controller designs.

Literature on system identification methodologies is quite rich [2], [3], [4]. For the first time, Nomoto [5] formulated the identification problem for ship dynamics. In his study, the model is linearized to possess some parameters that are easily identifiable with a zigzag test. Later on, Journee discussed parameter identification problem for steering dynamics of ships based on the same model [6]. As it has been followed in this study, parameter estimation technique is one of the most prevalent method in system identification, and it can be applied to USVs [7]. The general approach in parameter estimation is to minimize the error between true and predicted state histories with least squares regression.

Various other time-domain methods have been proposed for system identification of ship dynamics, such as Extended Kalman Filtering (EKF) [8] and adaptive back-stepping [9] on linearized models. Di Mascio et al. [10] proposed a method using Genetic Algorithm (GA). The performance of GA is shown to be superior over EKF based approaches under certain cases. Yoon and Rhee [11] used EKF and the Modified Bryson-Frazier Smoother (MBSF) to restore the time histories of the hy-

Authors thank to ASELSAN Inc. for their support. drodynamic forces. They concluded that the combination of a 20-20 degrees zigzag with 35 degrees turn manoeuvre was not rich enough to guarantee a reliable estimation. Perera et al. [12] utilized a stochastic parameter approach for an ocean vessel steering model with EKF. This study reached a conclusion that violent maneuvering conditions should be implemented in order to estimate the parameters of the nonlinear model accurately.

In addition to time-domain approaches, frequencydomain techniques have also been applied to USV parameter identification problem, yet still simple linear models are used, [13]. Tran et al. [14] have introduced an identification procedure to estimate the hydrodynamic parameters for a range of ships. This procedure used a dynamic ship motion model with mathematical programming techniques. The proposed methodology was validated through the turning and zigzag tests, and it was seen that there were improvements in root-mean square deviations of the ship trajectory and the heading angle. Kariotoglou [15] has proposed a methodology by using a planar motion mechanism to identify the pitch, roll and yaw damping parameters, and the linear drag parameters, and he validated his approach in simulations.

In this study, a system identification methodology is introduced to determine the parameters of USVs. A mathematical model for USVs is constructed, and the parameters to be identified are determined. A design of experiment approach is followed to construct a hierarchy in identification procedure. The experimental data in each test are post-processed, and identification routines are executed. In each step, the vehicle motion with predicted parameters are compared with experimental data. The parameters are tested in validation tests before proceeding with more complex motions. The final parameter set is validated in random motion tests. This system identifica- 
tion methodology to determine 6-DoF model parameters is the main contribution of this paper. The results show that the proposed method is quite successful in parameter identification.

\section{MATHEMATICAL MODELING}

A mathematical model expressing the motion of a surface vehicle in 6-DoF is built for this study. The model given by Eq. (1) is essentially adopted from [16].

$$
\begin{aligned}
& \dot{\eta}=\boldsymbol{J}_{\Theta}(\eta) \boldsymbol{v} \\
& \boldsymbol{M} \dot{\boldsymbol{v}}+\boldsymbol{C}(\boldsymbol{v}) \boldsymbol{v}+\boldsymbol{D}(\boldsymbol{v}) \boldsymbol{v}+\boldsymbol{g}(\eta)+\boldsymbol{g}_{0}=\tau+\tau_{d i s t}
\end{aligned}
$$

where linear positions and Euler angles are symbolized by $\eta \triangleq[x y z \phi \theta \psi]^{\mathrm{T}} \in \mathbb{R}^{3} \times \mathbb{S}^{3}$; linear and angular velocities of the body fixed reference frame, $\{b\}$, with respect to the earth fixed reference frame, $\{\mathrm{e}\}$, are denoted by $\boldsymbol{v} \triangleq[u v w p q r]^{\mathrm{T}} \in \mathbb{R}^{6}$. The transformation matrix, $\boldsymbol{J}_{\Theta}(\eta)$, is explicitly given in Eq. (2),

$$
\begin{aligned}
& {\left[\begin{array}{c}
\dot{\boldsymbol{p}}_{b \mid e}^{e} \\
\dot{\Theta}_{e b}
\end{array}\right]=\boldsymbol{J}_{\Theta}(\eta)\left[\begin{array}{c}
\boldsymbol{v}_{b \mid e}^{b} \\
\omega_{b \mid e}^{b}
\end{array}\right]} \\
& \boldsymbol{J}_{\Theta}(\eta)=\left[\begin{array}{cc}
\boldsymbol{R}_{b}^{e}\left(\Theta_{e b}\right) & \mathbf{0}_{3 \times 3} \\
\mathbf{0}_{3 \times 3} & \boldsymbol{T}_{\Theta}\left(\Theta_{e b}\right)
\end{array}\right]
\end{aligned}
$$

where $\boldsymbol{R}_{b}^{e}\left(\Theta_{e b}\right)$ is the Euler angle rotation matrix, and $\boldsymbol{T}_{\Theta}\left(\Theta_{e b}\right)$ is the angular velocity transformation matrix. Besides, $\boldsymbol{M}$ stands for the system inertial matrix; $\boldsymbol{C}(\boldsymbol{v})$ denotes the centrifugal and Coriolis matrix; $\boldsymbol{D}(\boldsymbol{v})$ refers to the hydrodynamic damping matrix; the buoyancy forces, $\boldsymbol{g}(\eta)$, and gravitational forces, $\boldsymbol{g}_{0}$, are together called restoring forces; $\tau$ represents thruster produced forces and moments; $\tau_{\text {dist }}$ stands for environmental disturbances [17]. In this study, the environmental disturbances are neglected.

The experimental part of this work is performed on a scale boat. A model Pacific Islander Tugboat measuring $900 \mathrm{~mm}$ in length, $290 \mathrm{~mm}$ in width and 260 $\mathrm{mm}$ in height, which is a $1 / 40$ scale of the actual vessel, is retrofitted with necessary sensors and computing units. Two independently-controllable propellers, which are driven by electric motors, are located at the back of the stern. The retrofitted vehicle, seen in Fig. 1, whose weight is about $11 \mathrm{~kg}$ can travel up to $2 \mathrm{~m} / \mathrm{s}$.

\section{SYSTEM IDENTIFICATION METHODOLOGY}

In this paper, gray-box, off-line, deterministic, time domain analysis is preferred as a system identification approach, according to the following remarks:

- Gray-box: High-fidelity mathematical model is developed.

- Offline: This kind of algorithms has higher performance compared to online ones. Real time optimized control action is not required.
- Deterministic: Raw data is post-processed with stochastic methods to increase accuracy.

- Time domain: Mathematical model is a nonlinear one. Frequency domain approach is not applicable.

Main steps of system identification methodology are shown in Fig. 2 and will be explained in following subsections.

\subsection{Determination of Parameters}

System identification procedure begins with determination of parameters to be identified. By careful investigation of mathematical model, it can be seen that there are twenty-three parameters to be estimated. These parameters can be grouped as follows: eight linear damping parameters $X_{u}, Y_{v}, Z_{w}, K_{p}, M_{q}, N_{r}, Y_{r}, N_{v}$, four inertia parameters $I_{x}, I_{y}, I_{z}, I_{x z}$, six nonlinear damping parameters $X_{u u}, Y_{v v}, Z_{w w}, K_{p p}, M_{q q}, N_{r r}$, four added mass parameters $X_{\dot{u}}, Y_{\dot{v}}, Y_{\dot{r}}, N_{\dot{r}}$ and one air drag parameter $C_{a d}$. Apart from mentioned parameters, there are four parameters left: $I_{y z}, I_{x y}, Y_{r}, N_{v}$, namely two inertia and two coupled damping parameters. Due to $x z$ plane symmetry of the ship, inertia parameters are taken to be zero. Furthermore, coupled damping parameters are assumed to be zero to reduce complexity.

\subsection{Design of Experiment}

The test procedures are determined by design of experiment approach. Literature related to design of experiments for parameter identification in surface vehicles proposes a variety of tests:

- Free motion tests for roll, pitch, yaw and heave movements

- Thrust measurement tests

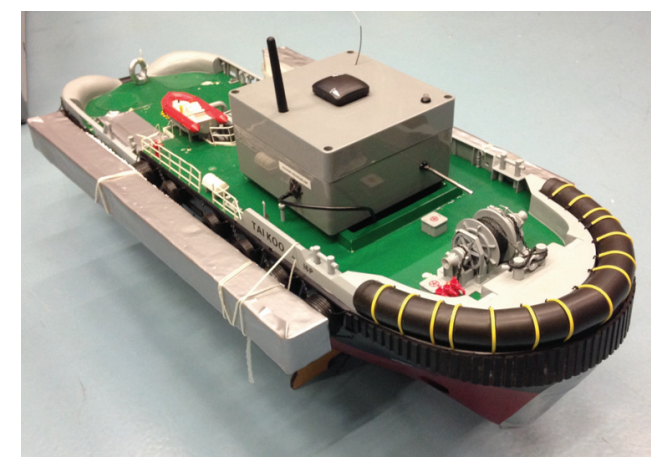

Fig. 1 Model Pacific Islander Tugboat with the installed equipments

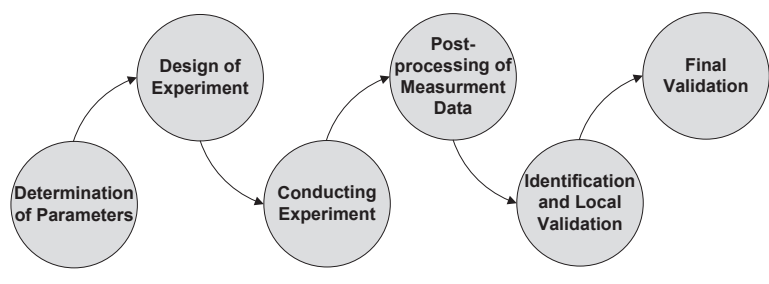

Fig. 2 System identification methodology 
- Linear motion tests

- Spiral maneuvering tests

- Zigzag maneuvering tests

The parameters that are effective in associated movements/experiments are determined by investigating the governing differential equations in the mathematical model. The equations are rewritten in symbolic notation of MATLAB ${ }^{\circledR}$ to elicit the unknown parameters and the states after substituting the other known terms. Backward-forward Euler method is preferred to acquire the derivatives of the states in the following form, as in Eq. (3).

$\dot{\boldsymbol{x}}=\boldsymbol{f}(\boldsymbol{x}, \boldsymbol{u}, \boldsymbol{P})$

where $\boldsymbol{x}$ is state vector, $\boldsymbol{u}$ is input vector that contains torque commands, and $\boldsymbol{P}$ are parameters to be identified. Note that Eq. (3) is just an abstract representation of Eq. (1).

Specifying appropriate test procedure and conducting experiments as accurate as possible are crucial steps in system identification. The details of experiments and the results are presented in Sec. 4.

\subsection{Post-Processing of Measurements}

After raw sensor measurement data are collected from various sensors, a post-processing is always required. These post-processing steps can be listed as follows:

- Generating a navigation solution

- Operation point transformation

Navigation solution determines the position, orientation and velocity of the vehicle. To this end, measurements from a gyroscope, an accelerometer, a GPS and a magnetometer are recorded throughout experiments. Then, a navigation algorithm is implemented to integrate inertial navigation solution (INS), GPS and attitude estimation in a loosely coupled, closed loop manner regarding errors included in these information sources such as biases, scale factor errors, cross coupling errors and random noises, [18]. The details of the navigation algorithm can be found in [19].

Next step is the operating point transformation. The mathematical model is established considering the quantities resolved in the center of gravity (CG) of the vehicle. On the other hand, since sensors are not necessarily located at CG, the navigation solution has to be transformed to $\mathrm{CG}$. This process is carried out by the system transformation matrix, $H\left(r_{p}^{b}\right)$, as given in [16].

\subsection{Identification with Local Verification}

The sub-methodology proposed in identification and local validation section is the novel part of this paper. Consisting of Initialization, Identification-Local Validation, and Final Validation steps, overview of this submethodology is presented in Fig. 3. Post-processed measurement data are used in optimization routines to identify the unknown parameters. The optimization routines

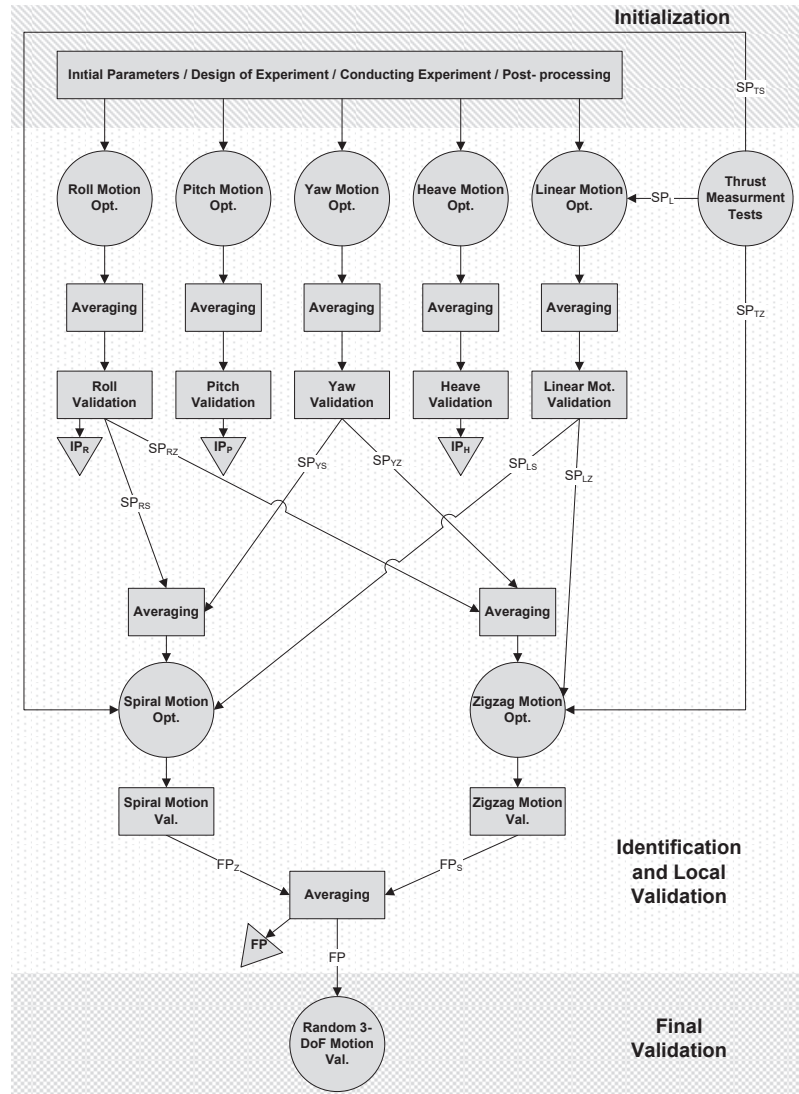

Fig. 3 Identification and Local Validation Methodology

take the initial values of unknown parameters, mathematical model, and algorithm parameters as inputs. Optimization infrastructure is shown in Fig. 4. In sum-

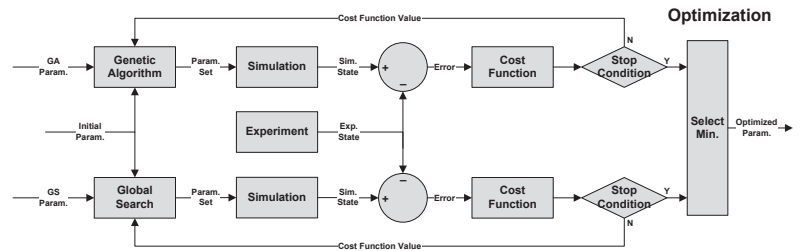

Fig. 4 Optimization structure

mary, a cost function defined as the integrated absolute error between the simulation results and the experimental measurements is tried to be minimized by using two different optimization algorithms (Genetic Algorithm (GA) and Global Search (GS)), and the algorithm yielding the minimum result is selected. Cost is least square regression type of function, and it is modified to handle the different length of test data easily. The reasons behind selection of optimization algorithms (GA and GS) and this type of cost function can be found in [20].

At the end of a optimization routine, as in Fig. 3, Isolated Parameters (IP), namely the parameters which are effective only in the performed motion test, reach their final values. However, Shared Parameters (SP), namely the parameters which are coupled into other tests, reach 
their final values after averaging the results from all the tests they affect. An estimate on a SP after one of these tests becomes the initialization point of the upcoming optimization routines. Local validation tests are performed in optimization routine with new batch data. If no new batch data is left for validation, the optimized parameters are validated with one of the available batches. It should be noted that subscripts of IP shown in Fig. 3 stand for the corresponding experiment (e.g., R: Roll motion test), and subscripts of SP demonstrate the feedback direction of estimates (e.g., RS: from roll to spiral motion test).

\subsection{Final Validation}

In final validation tests, identified parameters are validated in random 3-DoF motion tests in order to understand the effectiveness of the methodology. Final validation results are presented in Sec. 4.

\section{EXPERIMENTAL RESULTS}

The following procedure is valid for all free motion tests. It has resembling characteristics as the procedure given in [15].

- Sea surface vehicle is placed at the middle of the pool,

- Variety of initial conditions are provided with the help of external inclinometer and digital caliper,

- Data acquisition is initiated by an external computer,

- When the vehicle reaches stability, data acquisition is stopped.

\subsection{Roll Motion Test}

Initial condition of the vehicle for roll motion tests is depicted in Fig. 5. The vehicle is released to move freely

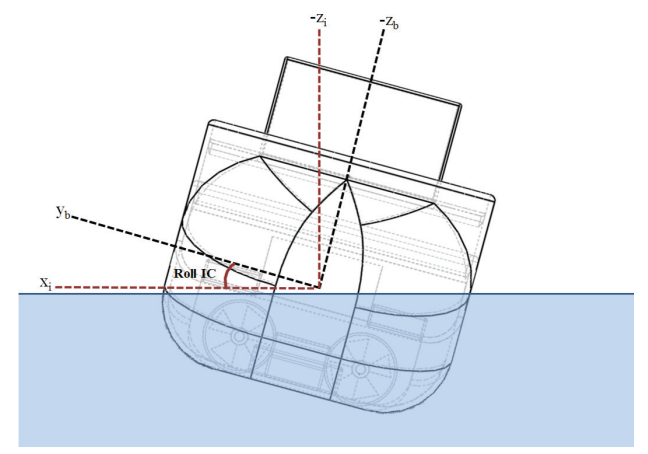

Fig. 5 Roll motion initial representation

around its $\mathrm{x}$-axes from the predetermined initial roll position. By neglecting surge, sway, heave, pitch and yaw motions, for roll motion tests the governing equation is obtained as in Eq. (4).

$$
\begin{aligned}
\dot{p}= & f_{a}\left(I_{x}, I_{z}, I_{x z}, Y_{\dot{v}}, N_{\dot{r}}, Y_{\dot{r}}\right) \phi+ \\
& f_{b}\left(I_{x}, I_{z}, I_{x z}, Y_{\dot{v}}, N_{\dot{r}}, Y_{\dot{r}}, K_{p}\right) p+ \\
& f_{c}\left(I_{x}, I_{z}, I_{x z}, Y_{\dot{v}}, N_{\dot{r}}, Y_{\dot{r}}, K_{p p}\right)|p| p \\
\dot{\phi}= & p
\end{aligned}
$$

As can be seen from Eq. (4), effective parameters in decoupled roll motion test are $I_{x}, I_{z}, I_{x z}, Y_{\dot{v}}, N_{\dot{r}}, Y_{\dot{r}}, K_{p}$ and $K_{p p}$. For optimization routines the cost function selected as given in Eq. (5).

RollCostFunc $=\sqrt{\frac{\sum_{j=1}^{N_{\text {point }}}\left(\phi_{i, \text { sim }}-\phi_{i, \text { test }}\right)^{2}}{N_{\text {point }}}}$

Four roll motion experiments are conducted. Three of them are utilized in identification and the remaining one is used for validation. The simulation outcome with the optimized parameters and its convergence to the measurement data are studied, and an example is depicted in Fig. 6.

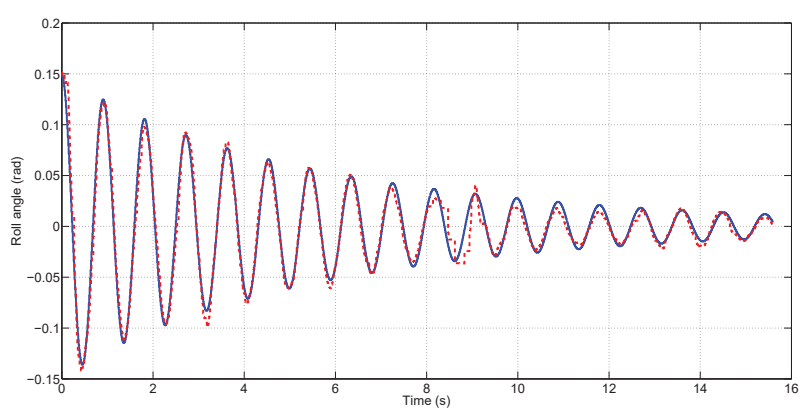

Fig. 6 Example of roll convergence: blue line for simulation results, red line for experimental data

Final optimized parameters, the average cost function value and the final cost value for validation can be found in [20]. Note that, in roll motion tests, $K_{p}$ and $K_{p p}$ are isolated parameters (IP), and $I_{x}, I_{z}, I_{x z}, Y_{\dot{v}}, N_{\dot{r}}, Y_{\dot{r}}$ are shared parameters (SP). The validation test results with simulation and experimental data are given in Fig. 7. As can be seen from the figure, convergence of the motion of the vehicle with the predicted parameters to the experimental data is quite sufficient. Similar tests are performed for pitch, yaw and heave motion experiments [20].

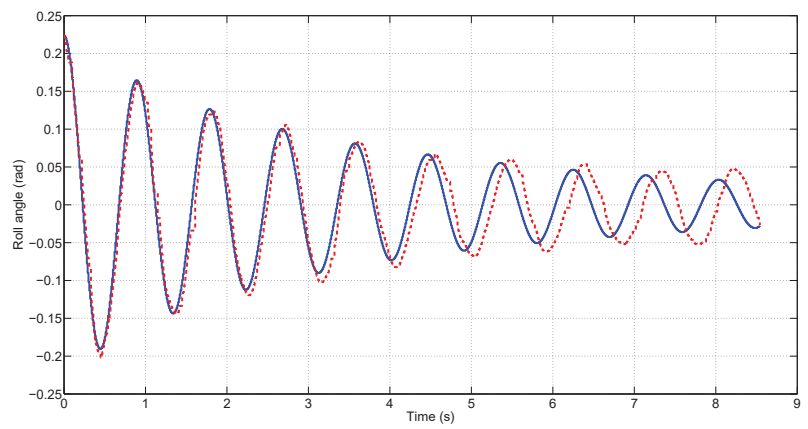

Fig. 7 Validation result for roll motion: blue line for simulation results, red line for experimental data

\subsection{Spiral Maneuvering Test}

In this test, surface vehicle follows a spiral-like path, and it is assumed that surge, sway and yaw motions are decoupled from the motions in other neglected axes. Similar to the approach in the previous test, differential equations are obtained for this coupled 3-DoF motion first. 
Surge, sway and yaw coupled motion equations are given in Eqs. (6), (7) and (8),respectively.

$$
\begin{aligned}
\dot{u}= & f_{a}\left(X_{u}, X_{\dot{u}}\right) u+f_{b}\left(X_{u u}, X_{\dot{u}}, C_{D A}\right)|u| u+ \\
& f_{c}\left(N_{\dot{v}}, Y_{\dot{r}}, X_{\dot{u}}\right) r^{2}+f_{d}\left(Y_{\dot{v}}, X_{\dot{u}}\right) r v+ \\
& f_{e}\left(X_{\dot{u}}\right) F_{l e f t}+f_{f}\left(X_{\dot{u}}\right) F_{r i g h t} \\
\dot{x}= & u \cos (\psi)-v \sin (\psi) \\
\dot{v}= & f_{a}\left(I_{x}, I_{z}, I_{x z}, N_{\dot{r}}, Y_{\dot{r}}, Y_{\dot{v}}, Y_{r}, N_{r}\right) r+ \\
& f_{b}\left(I_{x}, I_{z}, I_{x z}, N_{\dot{r}}, Y_{\dot{r}}, Y_{\dot{v}}, Y_{v}, N_{v}\right) v+ \\
& f_{c}\left(I_{x}, I_{z}, I_{x z}, N_{\dot{r}}, Y_{\dot{r}}, Y_{\dot{v}}, X_{\dot{u}}\right) r v+ \\
& f_{d}\left(I_{x}, I_{z}, I_{x z}, N_{\dot{r}}, Y_{\dot{r}}, Y_{\dot{v}}, Y_{v v}, C_{D A}\right)|v| v+ \\
& f_{e}\left(I_{x}, I_{z}, I_{x z}, N_{\dot{r}}, Y_{\dot{r}}, Y_{\dot{v}}, X_{\dot{u}}\right) u v+ \\
& f_{f}\left(I_{x}, I_{z}, I_{x z}, N_{\dot{r}}, Y_{\dot{r}}, Y_{\dot{v}}, N_{r r}\right)|r| r \\
\dot{y}= & v \cos (\psi)+u \sin (\psi) \\
\dot{\psi}= & f_{a}\left(I_{x}, I_{z}, I_{x z}, N_{\dot{r}}, Y_{\dot{r}}, Y_{\dot{v}}, Y_{r}, N_{r}\right) r+ \\
& f_{b}\left(I_{x}, I_{z}, I_{x z}, N_{\dot{r}}, Y_{\dot{r}}, Y_{\dot{v}}, Y_{v}, N_{v}\right) v+ \\
& f_{c}\left(I_{x}, I_{z}, I_{x z}, N_{\dot{r}}, Y_{\dot{r}}, Y_{\dot{v}}, X_{\dot{u}}\right) r u+ \\
& f_{d}\left(I_{x}, I_{z}, I_{x z}, N_{\dot{r}}, Y_{\dot{r}}, Y_{\dot{v}}, X_{\dot{u}}\right) u v+ \\
& f_{f}\left(I_{x}, I_{z}, I_{x z}, N_{\dot{r}}, Y_{\dot{r}}, Y_{\dot{v}}, N_{r r}\right)|r| r+ \\
& f_{g}\left(I_{x}, I_{z}, I_{x z}, N_{\dot{r}}, Y_{\dot{r}}, Y_{\dot{v}}, Y_{v v}, C_{D A}\right)|v| v+ \\
& f_{h}\left(I_{x}, I_{z}, I_{x z}, N_{\dot{r}}, Y_{\dot{r}}, Y_{\dot{v}}\right) F_{l e f t}+ \\
& f_{i}\left(I_{x}, I_{z}, I_{x z}, N_{\dot{r}}, Y_{\dot{r}}, Y_{\dot{v}}\right) F_{r i g h t}+ \\
\dot{\psi}= & r
\end{aligned}
$$

Combining all equations, parameters to be identified become $I_{x}, I_{z}, I_{x z}, X_{u}, X_{u u}, X_{\dot{u}}, Y_{v}, Y_{r}, Y_{v v}, Y_{\dot{r}}$, $Y_{\dot{v}}, N_{r}, N_{v}, N_{r r}, N_{\dot{r}}, C_{D A}, L_{T O}, L_{T S}, R_{T O}, R_{T S}$. The cost function is adopted to include $x, y$ and $\psi$ position errors.

Three spiral maneuvering tests are conducted. All of them are utilized for optimization and a randomly selected one is used for validation. The simulation outcome with the optimized parameters and its convergence to the measurement data are studied. An example result is presented in Fig. 8. Similar tests are performed for zig-zag motion experiments [20].

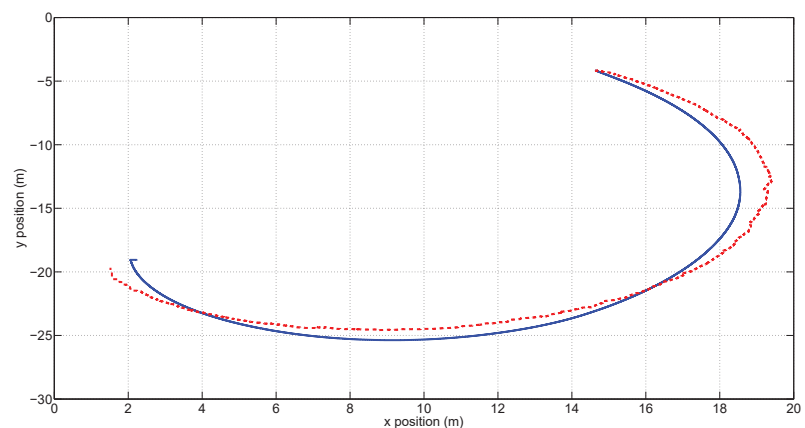

Fig. 8 Example of spiral convergence: blue line for simulation results, red line for experimental data

\subsection{Final Validation Tests}

Final values of the identified parameters are calculated by averaging of local estimates from spiral and zigzag motion tests, and they are listed in Table 1. As the next step, these results are validated on the two random motion tests. One of the validations is demonstrated in Figs. 9 and 10 .

Table 1 Final parameters

\begin{tabular}{|c|c|c|c|}
\hline$X_{u}$ & -5.6128 & $N_{r r}$ & -0.2605 \\
\hline$X_{u u}$ & -2.3136 & $N_{\dot{r}}$ & -0.0474 \\
\hline$X_{\dot{u}}$ & -1.0946 & $I_{x}$ & -2.5000 \\
\hline$Y_{v}$ & -3.5059 & $I_{z}$ & +0.0894 \\
\hline$Y_{r}$ & -0.0001 & $I_{x z}$ & +2.1493 \\
\hline$Y_{v v}$ & -3.9310 & $C_{D A}$ & +0.6487 \\
\hline$Y_{\dot{r}}$ & -1.4353 & $L_{T O}$ & +0.0228 \\
\hline$Y_{\dot{v}}$ & -1.0536 & $L_{T S}$ & +1.2466 \\
\hline$N_{r}$ & -0.1039 & $R_{T O}$ & -0.4907 \\
\hline$N_{v}$ & -1.0005 & $R_{T S}$ & +0.9730 \\
\hline \multicolumn{2}{|l|}{ Final Cost Function Value } & +0.7669 \\
\hline
\end{tabular}

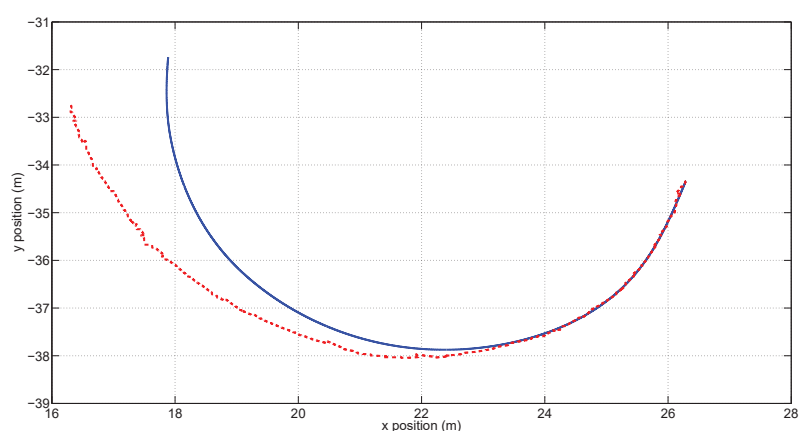

Fig. 9 Random motion path 1: blue line for simulation results, red line for experimental data
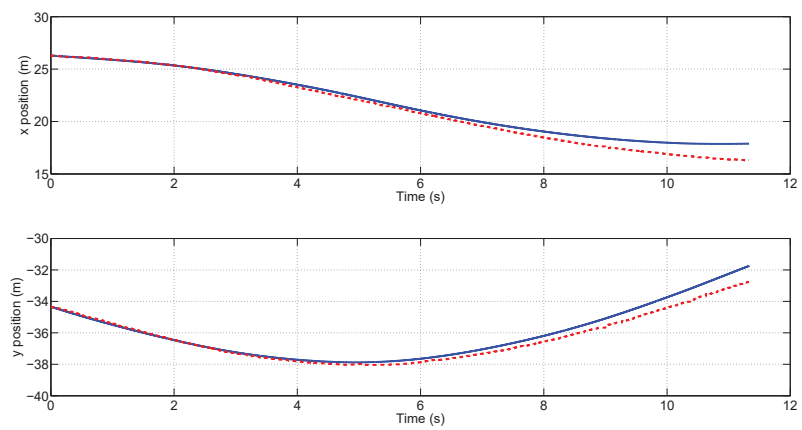

Fig. 10 Random motion path 1: blue line for simulation results, red line for experimental data

As can be seen from the results of final validation random motion tests, convergence of the motion with the predicted parameters to the experimental data is quite successful. It is an expected result, since the constructed mathematical model is not only a high fidelity model, but also flexible enough to be adopted for the free motion tests. Yet, there are still some small differences between the predicted and actual motions. Note that the experiments are not always conducted in a controlled medium. 
Environmental disturbance effects in each test can be different. Since the sea surface vehicle is relatively small, it is easily influenced by waves, wind and wind generated currents.

\section{CONCLUSION}

In this study, a system identification procedure is introduced to determine the parameters of unmanned surface vehicles. A mathematical model for USVs is constructed, and after a careful investigation, 23 parameters are observed to require identification to achieve a comprehensive understanding of the model behavior. According to the identification procedure, the tests are sequenced and the parameters are estimated. Optimization routines are initiated with a-priori information, and the costs between the vehicle motion with the predicted parameters and the experimental data are minimized. In a test, related parameters are identified and the results are validated. As the complexity of the motion increases, the effects of the coupled parameters are observed. The hierarchy proposed with the methodology elegantly handles this problem. The final parameter set is validated in random motion tests with rich motion content.

As future works, system identification results will be further validated with new experimental data. The effect of the navigation solution on the accuracy of the system identification procedure will be investigated. A stochastic system identification procedure can be considered if necessary, and the results can be compared with the ones obtained in this study.

\section{REFERENCES}

[1] K. Ahiska, "Control and guidance of an unmanned sea surface vehicle," Master's thesis, Dept. Elec. and Electronics Eng., Middle East Technical Uni., Turkey, 2012.

[2] L. Ljung, System Identification: Theory for the User. Prentice Hall information and system sciences series, Prentice Hall PTR, 1999.

[3] J. Juang, Applied System Identification. Mechanical engineering Aerospace, Prentice Hall, 1994.

[4] O. Nelles, Nonlinear system identification: from classical approaches to neural networks and fuzzy models. Springer-Verlag Berlin Heidelberg, 2001.

[5] K. Nomoto and K. Taguchi, "On steering qualities of ships (2)," Journal of Zosen Kiokai, vol. 1957, no. 101, pp. 57-66, 1957.

[6] J. Journee, "A simple method for determining the manoeuvring indices $\mathrm{k}$ and $\mathrm{t}$ from zigzag trial data," Report No: 0267, 1970.

[7] C. Sonnenburg, Modeling, identification and control of an unmanned surface vehicle. $\mathrm{PhD}$ thesis, Dept. Aerospace Eng., Virgina Polytechnic Institute and State Uni., USA, December 2012.

[8] W. Zhou and M. Blanke, "Nonlinear recursive pre- diction error method applied to identification of ship steering dynamics," in Eight Ship Control Systems Symposium, 1987.

[9] M. H. Casado, R. Ferreiro, and F. Velasco, "Identification of nonlinear ship model parameters based on the turning circle test," Journal of ship research, vol. 51, no. 2, pp. 174-181, 2007.

[10] A. Di Mascio, G. Dubbioso, C. Notaro, and M. Viviani, "Investigation of twin-screw naval ships maneuverability behavior," Journal of Ship Research, vol. 55, no. 4, pp. 221-248, 2011.

[11] H. K. Yoon and K. P. Rhee, "Identification of hydrodynamic coefficients in ship maneuvering equations of motion by estimation-before-modeling technique," Ocean Engineering, vol. 30, no. 18, pp. 2379 - 2404, 2003.

[12] L. P. Perera, P. Oliveira, and C. G. Soares, "System identification of vessel steering with unstructured uncertainties by persistent excitation maneuvers," IEEE Journal of Oceanic Engineering, vol. 41, pp. 515-528, July 2016.

[13] R. P. Selvam, S. Bhattacharyya, and M. Haddara, "A frequency domain system identification method for linear ship maneuvering," International shipbuilding progress, vol. 52, no. 1, pp. 5-27, 2005.

[14] K. T. Tran, A. Ouahsine, F. Hissel, and P. Sergent, "Identification of hydrodynamic coefficients from sea trials for ship maneuvering simulation," in Transport Research Arena (TRA) 5th Conference Transport Solutions from Research to Deployment, 2014.

[15] N. Kariotoglou, "Teer-by-wire of electric boats with two engines, modeling and control," Master's thesis, Swiss Federal Institute of Technology Zurich, 2015.

[16] T. I. Fossen, Handbook of Marine Craft Hydrodynamics and Motion Control. John Wiley \& Sons, Ltd, 2011.

[17] M. Kumru, I. Erunsal, K. Ahiska, and M. Leblebicioglu, "A survey on tactical control algorithms for path tracking unmanned surface vehicles," in 2016 14th International Conference on Control, Automation, Robotics and Vision (ICARCV), pp. 1-6, Nov 2016.

[18] P. D. Groves, Principles of GNSS, inertial, and multisensor integrated navigation systems. Artech house, 2013.

[19] M. Kumru, "Navigation and control of an unmanned sea surface vehicle," Master's thesis, Dept. Elec. and Electronics Eng., Middle East Technical Uni., Turkey, 2015.

[20] I. K. Erunsal, "System identification and control of a sea surface vehicle," Master's thesis, Dept. Elec. and Electronics Eng., Middle East Technical Uni., Turkey, 2015. 